POS $\quad$ PROCEEDINGS

\title{
The sextet gauge model, light Higgs, and the dilaton
}

\author{
Zoltán Fodor \\ Department of Physics, University of Wuppertal \\ Gaußstrasse 20, D-42119, Germany \\ Jülich Supercomputing Center, Forschungszentrum Jülich, D-52425 Jülich, Germany \\ Email: fodor@bodri.elte.hu
}

\section{Kieran Holland}

Albert Einstein Center for Fundamental Physics, Institute for Theoretical Physics, Bern University, Sidlerstrasse 5, CH-3012 Bern, Switzerland

Department of Physics, University of the Pacific, 3601 Pacific Ave, Stockton CA 95211, USA

Email: khollandepacific.edu

\section{Julius Kuti *}

Department of Physics 0319, University of California, San Diego

9500 Gilman Drive, La Jolla, CA 92093, USA

E-mail: jkuti@ucsd.edu

\section{Dániel Nógrádi}

Institute for Theoretical Physics, Eötvös University

H-1117 Budapest, Hungary

Email: nogradi@bodri .elte.hu

\section{Chris Schroeder}

Physical Sciences Directorate, Lawrence Livermore National Laboratory

Livermore, California 94550, USA

E-mail: schroeder10@llnl.gov

\section{Chik Him Wong}

Department of Physics 0319, University of California, San Diego

9500 Gilman Drive, La Jolla, CA 92093, USA

E-mail: rickywong@physics.ucsd.edu

The frequently discussed strongly interacting gauge theory with a fermion flavor doublet in the two-index symmetric (sextet) representation of the SU(3) color gauge group is investigated [1]. The chiral condensate and the mass spectrum are consistent with chiral symmetry breaking $(\chi \mathrm{SB})$ at vanishing fermion mass. In contrast, sextet fermion mass deformations of spectral properties are not consistent with leading conformal scaling behavior near the critical surface of a conformal theory. A recent paper [2] which could not resolve the conformal fixed point of the gauge coupling from the slowly walking scenario of a very small nearly vanishing $\beta$-function is not in conflict with $\chi \mathrm{SB}$ reported here. A light Higgs impostor could emerge as the dilaton from spontaneous symmetry breaking of scale invariance or, without the dilaton mechanism, as a composite state.

The 30 International Symposium on Lattice Field Theory - Lattice 2012, June 24-29, 2012

Cairns, Australia

${ }^{*}$ Speaker. 


\section{Introduction}

The new Higgs-like particle with decay modes not far from that of the Standard Model brings new focus and clarity to the search for theoretical frameworks. One example is the light dilaton as a pseudo-Goldstone particle of spontaneous breaking of scale invariance that has been featured in recent phenomenological discussions as a viable interpretation of the discovery. Nearly conformal gauge theories serve as theoretical laboratories for credible realizations of this scenario (this short report is based on [1] which provides references to the history of the field including recent work). We investigate here a candidate theory with a fermion flavor doublet in the two-index symmetric (sextet) representation of the $\mathrm{SU}(3)$ color gauge group close to the conformal window with a small beta function, as suggested by results of a recent paper [2]. With the limited statistical accuracy of the nearly vanishing $\beta$-function the existence of a conformal fixed point gauge coupling remains unresolved from the alternative slowly walking scenario. Consistency with $\chi \mathrm{SB}$, reported here for small fermion mass deformations, would require the sextet model to remain just below the conformal window with a very small non-vanishing $\beta$-function (see, also [3, 4]). In this case the model which exhibits the simplest composite Higgs mechanism leaves open the possibility of a light scalar state emerging as the pseudo-Goldstone dilaton state from spontaneous symmetry breaking of scale invariance. Even if scale symmetry breaking is entangled with $\chi \mathrm{SB}$ without dilaton interpretation, a light Higgs-like composite state can emerge close to the conformal window. We outline a new lattice Higgs project to resolve these important problems.

\section{Computational strategy and lattice simulations}

Probing $\chi \mathrm{SB}$, and conformal behavior for comparison, we extrapolate the spectrum to infinite volume at fixed fermion mass $m$. In large volumes the leading finite size corrections are exponentially small and dominated by the lowest state of the spectrum which is expected to have pion quantum numbers. From the mass spectrum, extrapolated to infinite volume, we can probe the pattern of $\chi \mathrm{SB}$ when small fermion mass deformations are simulated close to the massless limit. We also probe the hypothesis of mass deformed conformal scaling behavior and find results strongly favoring the $\chi \mathrm{SB}$ hypothesis.

A new analysis is presented at gauge coupling $\beta=3.2$ based on a subset of runs in the fermion mass range $\mathrm{m}=0.003-0.010$ on $24^{3} \times 48,28^{3} \times 56$, and $32^{3} \times 64$ lattices. Five fermion masses at $\mathrm{m}=0.003,0.004,0.005,0.006,0.008$ are used in most fits probing the two hypotheses. A very large and expensive $48^{3} \times 96$ run was added recently at $\mathrm{m}=0.003$ to control finite size effects. Simulation results at $\beta=3.25$ were also obtained in the mass range $\mathrm{m}=0.004-0.008$ on $24^{3} \times 48$, $28^{3} \times 56$, and $32^{3} \times 64$ lattices. We have used the tree-level Symanzik-improved gauge action with stout smeared staggered fermions for all results reported here. The normalization of the lattice coupling $\beta$ and details of the simulations are discussed in [1].

To control finite size effects, infinite-volume extrapolations were performed for the lowest state in the spectrum with pion quantum numbers, the related decay constant $F_{\pi}$, and the chiral condensate $\langle\bar{\psi} \psi\rangle$. They are shown in Figure 1 where $\widetilde{g}_{1}(\lambda, \eta)$ describes finite volume corrections from the exchange of the lightest pion state with $\lambda=M_{\pi} L$ and lattice aspect ratio $\eta=T / L$, similarly to what was introduced in [5]. The fitting procedure approximates the leading treatment of 

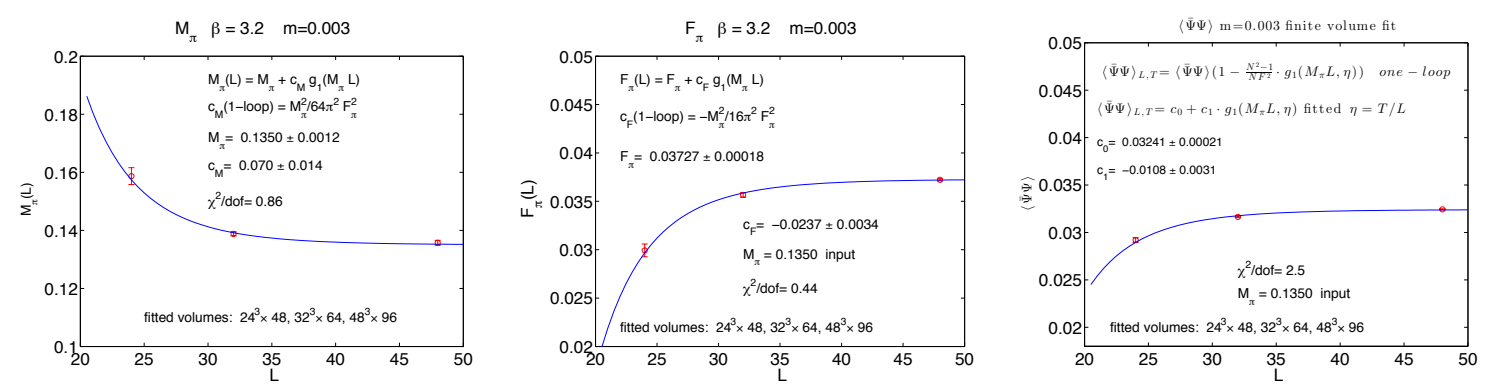

Figure 1: Finite volume dependence at the lowest fermion mass for $\beta=3.2$. The form of $\widetilde{g}_{1}(\lambda, \eta)$ is a complicated infinite sum which contains Bessel functions and requires numerical evaluation [7]. Since we are not in the chiral log regime, the prefactor of the $\widetilde{g}_{1}(\lambda, \eta)$ function was replaced by a fitted coefficient. The leading term of the function $\widetilde{g}_{1}(\lambda, \eta)$ is a special exponential Bessel function $K_{1}(\lambda)$ which dominates in the simulation range.

the pion which wraps around the finite volume, whether in chiral perturbation theory $(\chi \mathrm{PT})$, or in Lüscher's non-perturbative finite size analysis [6]. This equivalence relaxes the requirement on the fitted parameters $c_{M}, c_{F}, c_{1}$ to agree with 1-loop $\chi \mathrm{PT}$ as long as the pion is the lightest state dominating the finite volume corrections. The infinite-volume limits of $M_{\pi}, F_{\pi}$, and $\langle\bar{\psi} \psi\rangle$ for $m=0.003$ at $\beta=3.2$ were determined self-consistently from the fitting procedure. Similar fits were applied to other composite states. The value of $M_{\pi}$ in the fit of the left plot in Figure 1 was determined from the highly non-linear fitting function and used as input in the other two fits. Based on the fits at $m=0.003$, the results are within one percent of the infinite-volume limit at $M_{\pi} L=5$. In the fermion mass range $m \geq 0.004$ the condition $M_{\pi} L>5$ is reached at $L=32$ and we will interpret the results from the $32^{3} \times 64$ runs for $m \geq 0.004$ as infinite-volume behavior in mass deformed chiral and conformal analyses.

\section{The chiral condensate}

Our simulations show that the chiral condensate $\langle\bar{\psi} \psi\rangle$ is consistent with $\chi \mathrm{SB}$ and remains non-vanishing in the massless fermion limit. The cutoff dependent UV contributions are identified at finite fermion mass. The linear mass term $c_{1}(a) \cdot m$ is a quadratically divergent $\mathrm{UV}$ contribution $\approx a^{-2} \cdot m$ with lattice cutoff $a$. There is also a very small third-order UV term $c_{3}(a) \cdot m^{3}$ without power divergences which is hard to detect for small $m$ and has not been tested within the accuracy of the simulations. IR finite contributions are identified in the chiral expansion of the condensate. There is an $m$-independent constant term which is proportional to $B F^{2}$, a linear term proportional to $B^{2} \cdot m$, a quadratic term $\sim B^{3} F^{-2} \cdot m^{2}$, and higher order terms, in addition to logarithmic corrections generated from chiral loops. The expansion in the fermion mass is expressed in terms of low energy constants of chiral perturbation theory, like $B$ and $F$ [8]. A more complete description of our fitting functions is given in [1]. We used two independent methods for the determination of the chiral condensate in the massless fermion limit. In the first method fits were made directly to $\langle\bar{\psi} \psi\rangle$ with constant and linear terms in the fitted function. Quadratic and third order terms are hard to detect within the accuracy of the data. The result is shown in the left plot of Figure 2. When the quadratic term is added to the fit, the massless intercept $c_{0}=\langle\bar{\psi} \psi\rangle_{m=0}$ from the quadratic fit agrees with the one from the linear fit and the quadratic fit coefficient in $c_{2} \cdot \mathrm{m}^{2}$ is zero within fitting error. For an independent determination, we also studied the subtracted chiral condensate operator defined 

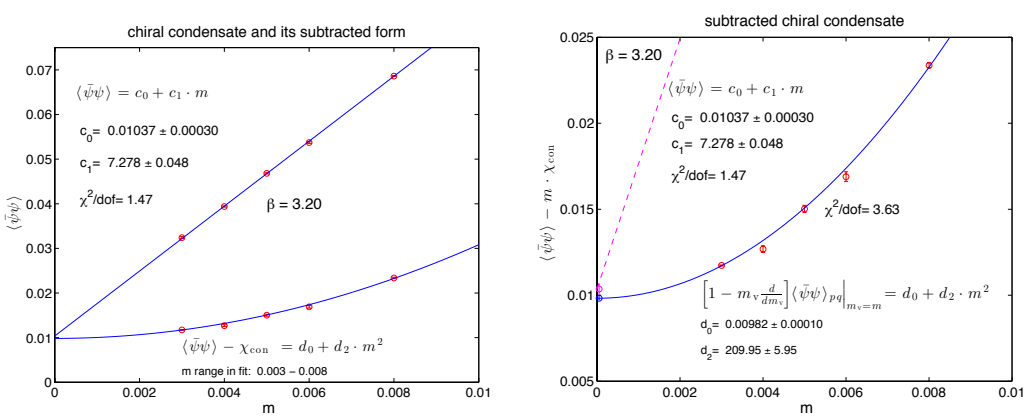

Figure 2: The chiral condensate and its reduced form with subtracted derivative (both have to converge to the same chiral limit) are shown in the left plot with linear fit to the condensate. The data without derivative subtraction cannot detect higher order fermion mass terms with significant accuracy. The fit to the reduced form with subtracted derivative is defined in [1] and shown in the magnified right plot. A linear term is not included in this fit since the subtracted derivative form approximately eliminates it. The value of $d_{0}$ at $m=0$ is shown to be consistent with the direct determination of $c_{0}$ from the chiral limit of $\langle\bar{\psi} \psi\rangle$. The consistency is very reassuring since the two results are derived from independent determinations. For $m=0.003$ the data from infinite-volume extrapolation were used in the fit. As we explained earlier, at higher $m$ values the largest volume $32^{3} \times 64$ runs were used for the condensate and its derivative subtraction.

with the help of the connected part $\chi_{\text {conn }}$ of the chiral susceptibility $\chi$. Once the derivative term is subtracted, the first non-perturbative IR contribution, quadratic in $m$, is better exposed. The two independent determinations give consistent non-vanishing fit results in the massless chiral limit as shown in Figure 2.

\section{Spectral tests of the $\chi \mathrm{SB}$ hypotheses}

The chiral Lagrangian describes the low energy theory of Goldstone pions and non-Goldstone pions in the staggered lattice fermion formulation. It can be used as an effective tool probing the $\chi$ SB hypothesis at finite fermion masses including extrapolation to the massless chiral limit. Close
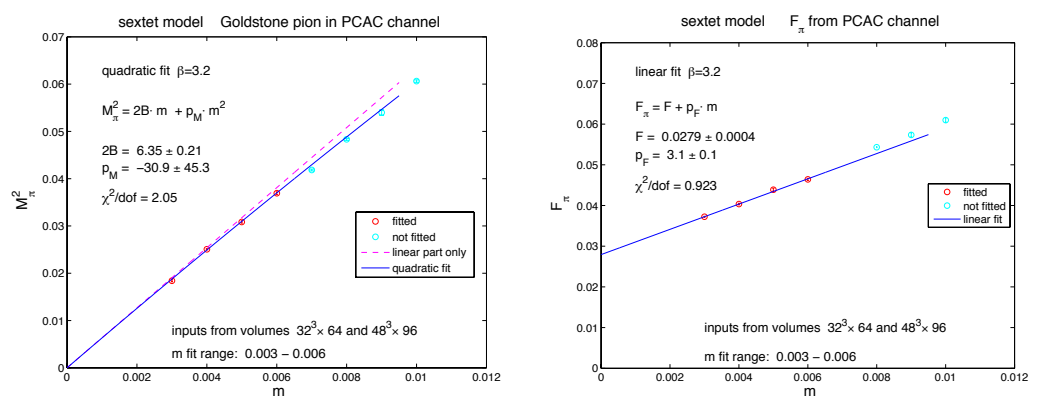

Figure 3: Polynomial fits from the analytic mass dependence of the chiral Lagrangian without logarithmic loop corrections are shown for the Goldstone pion and $F_{\pi}$. The dashed line in the left plot for the Goldstone pion shows the leading linear contribution. The data point at $m=0.003$ is determined from the $48^{3} \times 96$ lattice and the other fitted points are based on the $32^{3} \times 64$ runs.

to the chiral limit, the pion spectrum and the pion decay constant $F_{\pi}$ are organized in powers of the fermion mass $m$ which is an input parameter in the simulations. Chiral log corrections to the polynomial terms are generated from pion loops [9]. Their analysis will require an extended dataset with high statistics. 
In Section 2 we presented results of infinite-volume extrapolations. Based on these observations, in fits to the observed pion spectrum and $F_{\pi}$, we will use infinite-volume extrapolation at $m=0.003$ and treat the $32^{3} \times 64$ runs for $m \geq 0.004$ as if the volume were infinite. In Figure 3 we used the local pion correlator with noisy sources to extract $M_{\pi}$ and $F_{\pi}$. The correlator is tagged as the PCAC channel since the PCAC relation, based on axial Ward identities, holds for this correlator and the decay constant $F_{\pi}$ can be directly determined from the residue of the pion pole. Based on the analytic fermion mass dependence of the chiral Lagrangian, and using the lowest four fermion masses, good polynomial fits were obtained for $M_{\pi}$ and $F_{\pi}$ as shown in Figure 3 with fitting functions $M_{\pi}^{2}=2 B \cdot m+p_{M} \cdot m^{2}$ and $F_{\pi}=F+p_{F} \cdot m$. The parameters $B$ and $F$ are defined in the two leading terms of the chiral Lagrangian [9]. In this simple fitting procedure $B$ is set from the fit to $M_{\pi}^{2}$ and $F$ is set independently from the fit to $F_{\pi}$. The fit parameters $p_{M}$ and $p_{F}$ describe the respective leading power corrections in $m$ to the pion mass and decay constant. Limited to a single lattice spacing only, the accuracy of our dataset is not sufficient for the robust determination of chiral $\log$ corrections that will provide important consistency check for $B$ and $F$ in our future analysis of an extended dataset. This analysis requires rooted and partially quenched staggered perturbation theory at finite lattice spacing for simultaneous fits of $M_{\pi}$ and $F_{\pi}$ with a consistent pair of cutoff-dependent $F$ and $B$ values [10]. We made the first step in this direction by adding a new run set to our database at $\beta=3.25$. Reduction in taste breaking is significant at $\beta=3.25$ with smaller lattice spacing. Our staggered perturbation theory analysis will be presented elsewhere.

\section{Spectral tests of the conformal scaling hypothesis}

Under the conformal scaling hypothesis, the mass $M_{\pi}$ and the decay constant $F_{\pi}$ are given at leading order by $M_{\pi}=c_{M} \cdot m^{1 / 1+\gamma}$ and $F_{\pi}=c_{F} \cdot m^{1 / 1+\gamma}$. The coefficients $c_{M}$ and $c_{F}$ are channel specific but the exponent $\gamma$ is universal in all channels [11]. The leading scaling form sets in for small $m$ values, close to the critical surface. According to the hypothesis, there is an infrared conformal fixed point on the critical surface which controls the conformal scaling properties of small mass deformations. All masses of the spectrum can be subjected to similar conformal scaling tests, but we will mostly focus on accurate data in the $M_{\pi}$ and $F_{\pi}$ channels.

When $M_{\pi}$ and $F_{\pi}$ are fitted separately in the range of the four lowest fermion masses closest to the critical surface, we get reasonable $\chi^{2}$ values for the fits. However, the incompatibility of the fitted $\gamma$ values disfavors the hypothesis, inconsistent with mass deformed conformal behavior. The conflicting simultaneous fits to universal conformal form with the same $\gamma$ for the Goldstone
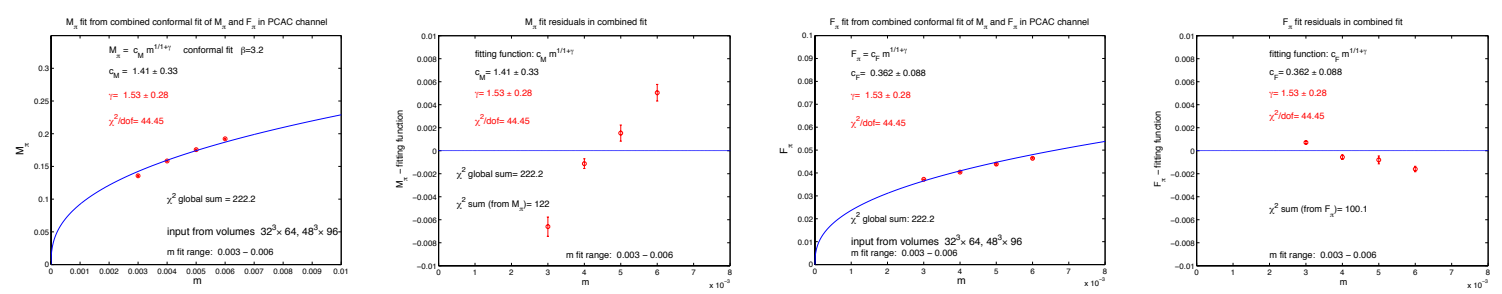

Figure 4: The first plot shows the simultaneous conformal fit result for the pion mass, while the second displays the $M_{\pi}$ residuals. The last two plots show the simultaneous fit result for the pion decay constant and the $F_{\pi}$ residuals. The combined fit forces $\gamma=1.53(28)$ with an unacceptable $\chi^{2} /$ dof of 44.5 . 
pion and the $F_{\pi}$ decay constant are illustrated in Figure 4. Fitting to the pion mass separately requires $\gamma=1.040$ (73) while the separate $F_{\pi}$ fit is forcing $\gamma=2.20(15)$. In the combined fit they compromise with $\gamma=1.53(28)$ and the unacceptable $\chi^{2} /$ dof of 44.5 . It is important to note that the exponent $\gamma$ for the fit to $M_{\pi}$ only is what $\chi \mathrm{SB}$ would prefer. The separate conformal exponent $\gamma$ for $F_{\pi}$ is large to force to the origin the linear string of data which extrapolate to a finite constant in $\chi \mathrm{SB}$. This creates conflict with the universal exponent $\gamma$ in the conformal analysis.

From the tests we were able to perform, the sextet model is consistent with $\chi \mathrm{SB}$ in leading approximation and inconsistent with conformal symmetry. It will require further investigations to show that subleading effects cannot alter this conclusion. It is particularly important to strengthen the analysis of the $\chi$ SB hypothesis reaching the necessary accuracy to identify chiral log effects. We will also consider comprehensive conformal finite size scaling (FSS) tests which do not rely on infinite-volume extrapolation in the scaling fits. It remains difficult to reconcile $\chi \mathrm{SB}$ and large exponents in the fermion mass dependence with the low value of $\gamma$ defined by the chiral condensate using the Schödinger functional for massless fermions [2].

\section{The new sextet Higgs project}

Figure 5 shows the fermion mass dependence of the scalar $0^{++}\left(f_{0}\right)$ meson without including the disconnected part of correlator I in Table 1 of [12]. The non-Goldstone scPion and $f_{0}$ are opposite parity states in this staggered correlator. The quantum numbers of the $f_{0}$ meson match those of the $0^{++}$state in the staggered correlator. Close to the conformal window the scalar $0^{++}$ meson is not expected to be similar to the $\sigma$ particle of QCD. If it turns out to be light, the full scalar state, including the disconnected gluon annihilation diagram and mixing with the $0^{++}$glueball state, could replace the role of the elementary Higgs and act as the Higgs impostor. It is very difficult to do the full calculation including the disconnected gluon annihilation diagram and mixing with the $0^{++}$glueball state. This is the main part of our next generation sextet Higgs project.

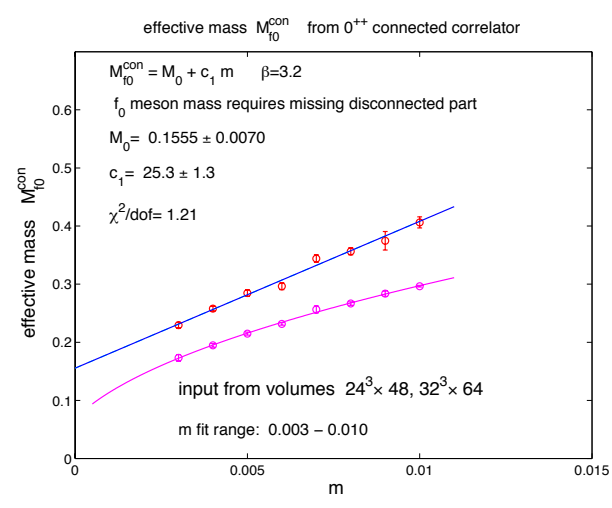

Figure 5: The linear fit is shown to the mass of the scalar $0^{++}\left(f_{0}\right)$ meson from the connected part of correlator I in Table 1 of [12]. For comparison, the scPion which is the opposite parity state in the staggered correlator is also plotted (at $m=0$ the mass of the non-Goldstone scPion will vanish in the continuum). Gluon annihilation and mixing of the $f_{0}$ state with a low mass $0^{++}$glueball state could generate a light scalar mass close to the conformal window. The disconnected part of the staggered correlator and its mixing with the glueball correlator are required to resolve this issue.

The linear fit from the connected diagram is shown in Figure 5. It has a non-zero intercept in the chiral limit with a mass more than five times $F$ so it corresponds to a heavy state and not a light 
Higgs candidate. Close to the conformal window it is reasonable to expect that the disconnected diagram, after mixing with the $0^{++}$glueball state will lead to a light scalar mass in $F$ units when the chiral limit is taken. The light scalar state after mixing remains a viable Higgs candidate. Only new simulations can resolve the issue and perhaps eliminate this attractive scenario.

To study flavor-singlet mesons, we need to consider fermion loops which are disconnected (often called hairpin diagrams). Flavor-singlet correlators have fermion-line connected and fermionline disconnected contributions from the hairpin diagrams. To evaluate disconnected quark loops with zero momentum, we need to sum over propagators from sources at each spatial location for a given time slice. To avoid the very costly $\mathscr{O}(V)$ inversions to compute all-to-all propagators in lattice terminology, random sources have to be used with noise reduction.

A very interesting remaining challenge and complication is the existence of two types of distinct $0^{++}$scalar states. One of them is the composite meson state and the other is the scalar glueball with the same $0^{++}$quantum numbers. In dynamical sextet simulations, these two types of state will mix producing two scalar states. To resolve this will require a well-chosen variational operator set with room left for a light scalar state to emerge in the spectrum. It is also entirely possible that careful lattice calculations will shut down the light Higgs interpretation.

\section{Acknowledgments}

We acknowledge support by the DOE under grant DE-FG02-90ER40546, by the NSF under grants 0704171 and 0970137, by the EU Framework Programme 7 grant (FP7/2007-2013)/ERC No 208740, and by the Deutsche Forschungsgemeinschaft grant SFB-TR 55. Computational resources were provided by USQCD at Fermilab and JLab, by the NSF XSEDE program, and by the University of Wuppertal. KH wishes to thank the Institute for Theoretical Physics and the Albert Einstein Center for Fundamental Physics at Bern University for their support. KH and JK wish to thank the Galileo Galilei Institute for Theoretical Physics and INFN for their hospitality and support at the workshop "New Frontiers in Lattice Gauge Theories".

\section{References}

[1] Z. Fodor, K. Holland, J. Kuti, D. Nogradi, C. Schroeder and C. H. Wong, Phys. Lett. B 718, 657 (2012).

[2] T. DeGrand, Y. Shamir and B. Svetitsky, arXiv:1201.0935 [hep-lat].

[3] J. B. Kogut, D. K. Sinclair, Phys. Rev. D81, 114507 (2010).

[4] J. B. Kogut and D. K. Sinclair, Phys. Rev. D 84, 074504 (2011) [arXiv:1105.3749 [hep-lat]].

[5] H. Leutwyler, Phys. Lett. B 189, 197 (1987).

[6] M. Luscher, Commun. Math. Phys. 104, 177 (1986).

[7] J. Gasser, H. Leutwyler, Phys. Lett. B184, 83 (1987).

[8] J. Bijnens, J. Lu, JHEP 0911, 116 (2009). [arXiv:0910.5424 [hep-ph]].

[9] J. Gasser and H. Leutwyler, Annals Phys. 158, 142 (1984).

[10] C. Aubin and C. Bernard, Phys. Rev. D 68, 034014 (2003).

[11] L. Del Debbio, R. Zwicky, Phys. Rev. D82, 014502 (2010).

[12] N. Ishizuka, M. Fukugita, H. Mino, M. Okawa, A. Ukawa, Nucl. Phys. B411, 875-902 (1994). 